\title{
Transient severe motion during arterial phase in patients with Gadoxetic acid administration: Can a five hepatic arterial subphases technique mitigate the artifact?
}

\author{
YU-DONG XIAO, CONG MA, JUN LIU, HUA-BING LI, SHUN-KE ZHOU and ZI-SHU ZHANG
}

Department of Radiology, The Second XiangYa Hospital of Central South University, Changsha, Hunan 410011, P.R. China

Received August 15, 2017; Accepted January 4, 2018

DOI: $10.3892 / \mathrm{etm} .2018 .5760$

\begin{abstract}
Gadoxetic acid (Gd-EOB-DTPA) is a hepatocyte-specific magnetic resonance (MR) contrast agent, which has been increasingly used in recent years. However, it has been reported that Gd-EOB-DTPA related transient severe motion (TSM) is sometimes observed during the hepatic arterial phase of MR imaging, which may influence image quality. Since the hepatic arterial phase of contrast enhancement is used for the diagnosis of hepatocellular carcinoma, it is crucial to obtain a decent arterial phase imaging. The present study analyzed motion in patients receiving Gd-EOB-DTPA, comparing a single arterial phase acquisition to a five arterial phase acquisition to determine whether the multiphase acquisition was able to alleviate the TSM-related hepatic arterial MR imaging artifact. It was demonstrated that the single-phase acquisition failed to provide adequate diagnostic image quality in patients with TSM, whereas the multiphase arterial acquisition provided acceptable image quality in 20/22 (90.9\%) patients with TSM. In conclusion, the results of the present study demonstrated that multiphase arterial acquisition is superior to single-phase arterial acquisition, mitigating arterial MR imaging artifacts caused by TSM after the administration of Gd-EOB-DTPA.
\end{abstract}

Correspondence to: Professor Zi-Shu Zhang, Department of Radiology, The Second XiangYa Hospital of Central South University, 139 Middle Renmin Road, Changsha, Hunan 410011, P.R. China E-mail: zishuzhang@hotmail.com

Abbreviations: Gd-EOB-DTPA, gadoxetic acid; MR, magnetic resonance; HCC, hepatocellular carcinoma; HBP, hepatobiliary phase; FLL, focal liver lesion; TSM, transient severe motion; VIBE, volume interpolated breath-hold examination; CAIPIRINHA, controlled aliasing in parallel imaging results in higher acceleration; TWIST, time-resolved imaging with interleaved stochastic trajectories; CDT-VIBE, CAIPIRINHA-Dixon-TWIST-VIBE; ICC, intraclass correlation coefficient; BMI, body mass index

Key words: gadoxetic acid, magnetic resonance, arterial phase, artifact, hepatocellular carcinoma

\section{Introduction}

Gadoxetic acid (Gd-EOB-DTPA) is a hepatocyte-specific magnetic resonance (MR) contrast agent $(1,2)$, which has being used increasingly in recent years for imaging diagnosis of hepatocellular carcinoma (HCC), liver metastasis, and other diseases (3-7). Its advantage lies on the addition of offering hepatobiliary phase (HBP) imaging (8). After intravenous injection, Gd-EOB-DTPA is gradually taken up by functional hepatocyte and eventually excreted via biliary and renal system $(9,10)$. Owing to this property, Gd-EOB-DTPA has been thought to be superior to the conventional extracellular Gd-based contrast agents, especially in the early detection of focal liver lesions (FLLs) (11-13). However, despite the advantages of HBP imaging in Gd-EOB-DTPA-enhanced $\mathrm{MR}$, arterial phase enhancement remains critical for the detection and characterization of FLLs (14). In previous study, it has been reported that transient severe motion (TSM) often occurred after administration of Gd-EOB-DTPA (15). Our previous study showed that TSM is more often observed in patients receiving Gd-EOB-DTPA than those receiving Gd-DTPA (16). TSM has been described by patients as a temporary, self-limiting phenomenon that lasts about $10-20 \mathrm{sec}$. The mechanism of such phenomena still remains unclear, and some authors have attributed its severity to Gd-EOB-DTPA-related dose-toxicity $(17,18)$. In order to minimize the issue related TSM, a body-weight calibrated dose and a slower injection rate of contrast has been adopted in previous studies $(14,17)$. Since TSM may have a serious effect on image quality during the arterial phase, and affect diagnostic accuracy of liver disease, it is crucial to provide optimal arterial phase imaging.

Recently, a newly developed technique introduced by Michaely et al (19), has been shown to significantly increase the temporal resolution during multiphasic imaging. This technique has demonstrated that within a single breath-hold, in combination of volume interpolated breath-hold examination (VIBE) with controlled aliasing in parallel imaging results in higher acceleration (CAIPIRINHA), view-sharing time-resolved imaging with interleaved stochastic trajectories (TWIST), and Dixon fat suppression (CAIPIRINHA-Dixon-TWIST-VIBE, CDT-VIBE), is capable of obtaining the high spatial and temporal resolution of multiple hepatic arterial subphases $(19,20)$. To the best of our knowledge, there is no published research utilizing such 
a technique in order to investigate TSM on arterial phase imaging.

This study, therefore, aims to investigate the comparison between patient receiving single phase of VIBE (single-VIBE) technique and those receiving five subphases of CDT-VIBE (5-CDT-VIBE) technique during arterial phase with Gd-EOB-DTPA, and whether 5-CDT-VIBE technique can provide adequate diagnostic information in patients with TSM.

\section{Materials and methods}

Patients. This retrospective study was approved by the ethics committee of the Second Xiangya Hospital of Central South University. All clinical investigations were conducted according to the principles of the Declaration of Helsinki. Written informed consent was obtained from all patients before inclusion.

From January 2013 to March 2016, a total of consecutive 463 patients (310 males and 153 females) who were the first time suggested undergoing Gd-EOB-DTPA-enhanced MR of the liver with known or unknown liver lesions were included in the study. Eighty-five patients who were referred for the evaluation of the liver with Gd-EOB-DTPA-enhanced MRI of the liver did not undergo the requested study because they met the exclusion criteria: known pulmonary disease (which may influence the breath holding) such as chronic obstructive pulmonary disease (COPD), pleural effusion, and MR examination contraindication such as known renal inadequacy (glomerular filtration rate, GFR $<30 \mathrm{ml} / \mathrm{min}$ ), existence of claustrophobia, post-implantation of pacemaker, breath holding time less than $20 \mathrm{sec}$, and related adverse reaction of gadolinium chelates. Finally, the total study population comprised 378 patients (239 males, 139 females, ranged in age from 26-85 years with a mean age of $57.3 \pm 18.2$ years), which was consisted of HCC $(n=170)$, liver metastasis $(n=95)$, focal nodular hyperplasia $(\mathrm{FNH}, \mathrm{n}=45)$, cholangiocellular carcinoma $(\mathrm{CCC}, \mathrm{n}=32)$, hemangioma $(n=17)$, hepatocellular adenoma, $(\mathrm{HCA}, \mathrm{n}=13)$, regenerative nodules in liver cirrhosis $(\mathrm{RN}, \mathrm{n}=6)$. The final diagnosis of the liver lesions was based on either histopathology (resection, $n=112$; biopsy, $n=46$ ), the 2014 version of Liver Imaging Reporting and Data System (LI-RADS) criteria $(n=146)$, or existence of primary extrahepatic tumor $(n=74)$. The potential risk factors that might have an influence on the respiratory motion artifact were recorded, which included age, gender, body mass index (BMI), Child-Pugh grading, and the relative volume of ascites. The relative volume of ascites was scored according to the imaging qualitative scale of $1-3,1$ was classified as absent of ascites, 2 was classified as small amount of ascites, and 3 was classified as moderate to large amount of ascites. The detailed demographic of all patients is listed in Table I.

MR imaging technique. MR imaging was performed under a clinical 3.0 Tesla superconducting MR system (Magnetom Skyra; Siemens Healthcare, Germany) with the 18-channel body matrix coil and the inbuilt 24-channel spine matrix coil. The comprehensive MRI protocol including T1-weighted fat saturation gradient recalled echo sequence as well as T2-weighted half fourier acquisition single-shot turbo spin-echo sequence were obtained prior to Gd-EOB-DTPA
Table I. Demographic data for all patients.

\begin{tabular}{lccc}
\hline Factor & All & Single-VIBE & 5-CDT-VIB \\
\hline Age, years & $57.3 \pm 18.2$ & $53.1 \pm 14.2$ & $59.8 \pm 19.4$ \\
Sex & & & \\
Male & 239 & 135 & 104 \\
Female & 139 & 81 & 58 \\
Etiology & & & \\
HCC & 170 & 98 & 72 \\
Liver metastasis & 95 & 53 & 42 \\
FNH & 45 & 25 & 20 \\
CCC & 32 & 17 & 15 \\
Hemangioma & 17 & 11 & 6 \\
HCA & 13 & 8 & 5 \\
RN & 6 & 4 & 2 \\
\hline
\end{tabular}

HCC, hepatocellular carcinoma; FNH, focal nodular hyperplasia; CCC, cholangiocellular carcinoma; HCA, hepatocellular adenoma; $\mathrm{RN}$, regenerative nodule; Single-VIBE, single-volume interpolated breath-hold examination; 5-CDT-VIBE, 5-CAIPIRINHA-Dixontime-resolved imaging with interleaved stochastic trajectories-VIBE.

administration. Among 378 patients, either 5-CDT-VIBE technique $(n=162)$ or single-VIBE technique $(n=216)$ was performed on the unenhanced phase, arterial phase, portovenous phase ( $90 \mathrm{sec})$, the delayed phase (180 sec), and the HBP (20 min). In order to catch the same contrast-enhanced phase in every patient, a MR automated injector pump was used to administer Gd-EOB-DTPA through an 18-gauge cubital intravenous access at a dose of $0.1 \mathrm{ml} / \mathrm{kg}$ body weight and an injection rate of $1 \mathrm{ml} / \mathrm{s}$. The 5-CDT-VIBE of initial arterial phase started at a standard timing of $18 \mathrm{sec}$ after the start of contrast agent injection with a temporal resolution of $2.6 \mathrm{sec}$. The detailed MR parameters are listed in Table II.

Imaging analysis. Two radiologists with 20-year and 12-year liver imaging experience independently and randomly reviewed all images. Both radiologists were blinded to the clinical data and each other's results. Respiratory motion artifact was evaluated using a scoring system, as previously described, with high reproducibility (15). The degree of respiratory motion artifact was scored from 1 to 5, 1 was defined as no motion artifact, 2 was defined as minimal motion artifact without effect on diagnostic quality, 3 was defined as moderate motion artifact, with some, but not severe effect on diagnostic quality, 4 was defined as severe motion artifact, but images still interpretable, and 5 was defined as extensive motion artifact and images non-diagnostic quality. The scoring system and the typical imaging of motion artifact are presented in Fig. 1. TSM was defined as motion score 2 or less on unenhanced phase (or every one of motion score on 5 unenhanced subphases), meanwhile motion score 4 or more on arterial phase (or any one of motion score on 5 arterial subphases). The average motion score between two radiologists was calculated in each subphase.

Statistical analysis. The intraclass correlation coefficients (ICCs) were calculated to evaluate the inter-observer 
Table II. MR sequence parameters.

\begin{tabular}{lccc}
\hline Parameters & Single-VIBE & 5-CDT-VIBE & T2 HASTE \\
\hline TR/TE, milliseconds & $2.9 / 1.4$ & $3.8 / 1.2$ & $2000 / 80$ \\
Subphases & 1 & 5 & 1 \\
Sequence type & VIBE & TWIST-VIBE & HASTE \\
Voxel size, mm $^{3}$ & $1.3 \times 1.3 \times 3.0$ & $1.3 \times 1.3 \times 3.0$ & $1.3 \times 1.3 \times 3.0$ \\
FOV, mm & 380 & 380 & 380 \\
Slice number & 72 & 72 & 72 \\
Flip angle, degree & 9 & 9 & 180 \\
Respiratory control & Breath hold & Dreath hold & Triggered \\
Fat suppression & Spectral saturation & 2.6 & Spectral saturation \\
Temporal resolution, $s$ & - & 20 & - \\
TWIST size of k-center, $\%$ & - & 25 & - \\
TWIST size of k-periphery, $\%$ & - & 20 & - \\
Breath holding time, seconds & $9-22$ & $10-15$ & \\
\hline
\end{tabular}

T, repetition time; TE, echo time; VIBE, volume-interpolated breath hold examination; HASTE, half-Fourier acquisition single-shot turbo spin-echo; TWIST, time-resolved imaging with interleaved stochastic trajectories; FOV, field of view.

Table III. Patient characteristics and risk factors in patients with or without TSM.

\begin{tabular}{|c|c|c|c|c|c|c|c|c|c|}
\hline \multirow[b]{2}{*}{ Characteristic } & \multicolumn{3}{|c|}{ Single-VIBE } & \multicolumn{3}{|c|}{ 5-CDT-VIBE } & \multicolumn{3}{|c|}{ Overall } \\
\hline & TSM & Non-TSM & P-value & TSM & Non-TSM & P-value & TSM & Non-TSM & P-value \\
\hline BMI & $25.1 \pm 2.1$ & $19.7 \pm 2.6$ & 0.03 & $27.1 \pm 2.5$ & $20.4 \pm 1.8$ & 0.03 & $26.7 \pm 3.4$ & $19.8 \pm 4.5$ & 0.02 \\
\hline Age, years & $52.7 \pm 13.7$ & $56.3 \pm 16.4$ & 0.47 & $60.1 \pm 17.7$ & $57.6 \pm 19.6$ & 0.39 & $58.5 \pm 17.1$ & $56.4 \pm 19.5$ & 0.45 \\
\hline Child grading, $\%$ & 25 & 191 & 0.87 & 22 & 140 & 0.35 & 47 & 331 & 0.84 \\
\hline A & $13(52)$ & $108(56.5)$ & - & $8(36.4)$ & $74(52.9)$ & - & $21(44.7)$ & $162(48.9)$ & - \\
\hline $\mathrm{B}$ & $9(36)$ & $59(30.9)$ & - & $10(45.4)$ & $48(34.3)$ & - & $19(40.4)$ & $127(38.4)$ & - \\
\hline $\mathrm{C}$ & $3(12)$ & $24(12.6)$ & - & $4(18.2)$ & $18(12.8)$ & - & 7 (14.9) & $42(12.7)$ & - \\
\hline Ascites grading, $\%^{a}$ & 25 & 191 & $<0.01$ & 22 & 140 & $<0.01$ & 47 & 331 & $<0.01$ \\
\hline 1 & $8(32)$ & $143(74.9)$ & - & $7(31.8)$ & 97 (69.3) & - & $15(31.9)$ & $240(72.5)$ & - \\
\hline 2 & $7(28)$ & $29(15.2)$ & - & $5(22.7)$ & 29 (20.7) & - & $12(25.5)$ & $58(17.5)$ & - \\
\hline 3 & $10(40)$ & $19(9.9)$ & - & $10(45.5)$ & $14(10)$ & - & $20(42.6)$ & $33(10)$ & - \\
\hline Sex, \% & 25 & 191 & 0.78 & 22 & 140 & 0.68 & 47 & 331 & 0.93 \\
\hline Male & $15(60)$ & $120(62.8)$ & - & $15(68.2)$ & 89 (63.6) & - & $30(63.8)$ & $209(63.1)$ & - \\
\hline Female & $10(40)$ & $71(37.2)$ & - & $7(31.8)$ & $51(36.4)$ & - & $17(36.2)$ & $122(36.9)$ & - \\
\hline
\end{tabular}

${ }^{\mathrm{a}} 1$ was classified as absent of ascites, 2 was classified as small amount of ascites, and 3 was classified as moderate to large amount of ascites. BMI, body mass index; TSM, transient severe motion; Single-VIBE, single-volume interpolated breath-hold examination; 5-CDT-VIBE, 5-CAIPIRINHA-Dixon-time-resolved imaging with interleaved stochastic trajectories-VIBE.

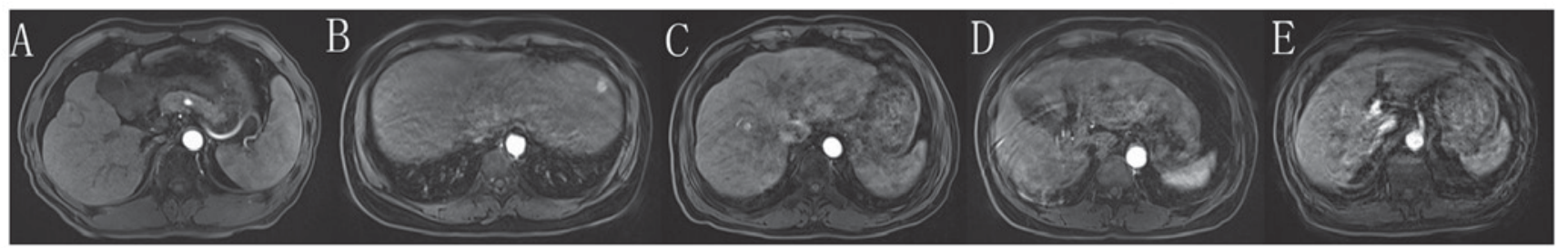

Figure 1. Typical imaging of motion scores. (A) Motion score of 1: No motion artifact; (B) motion score of 2: Minimal motion artifact without effect on diagnostic quality; (C) motion score of 3: Moderate motion artifact, with some, but not severe effect on diagnostic quality; (D) motion score of 4: Severe motion artifact, but images still interpretable; (E) motion score of 5: Extensive motion artifact and images non-diagnostic quality. 
Table IV. ICCs between two reviewers.

\begin{tabular}{|c|c|c|c|c|c|c|c|c|c|c|}
\hline \multirow[b]{2}{*}{ Technique } & \multicolumn{5}{|c|}{ Unenhanced subphases } & \multicolumn{5}{|c|}{ Arterial subphases } \\
\hline & First & Second & Third & Fourth & Fifth & First & Second & Third & Fourth & Fifth \\
\hline $\begin{array}{l}\text { 5-CDT-VIBE } \\
\text { Single-VIBE }\end{array}$ & 0.86 & 0.91 & $\begin{array}{l}0.74 \\
0.91\end{array}$ & 0.88 & 0.93 & 0.89 & 0.71 & $\begin{array}{l}0.68 \\
0.85\end{array}$ & 0.78 & 0.87 \\
\hline
\end{tabular}

ICC, intraclass correlation coefficient; 5-CDT-VIBE, 5-CAIPIRINHA-Dixon-time-resolved imaging with interleaved stochastic trajectories-VIBE; Single-VIBE, single-volume interpolated breath-hold examination.

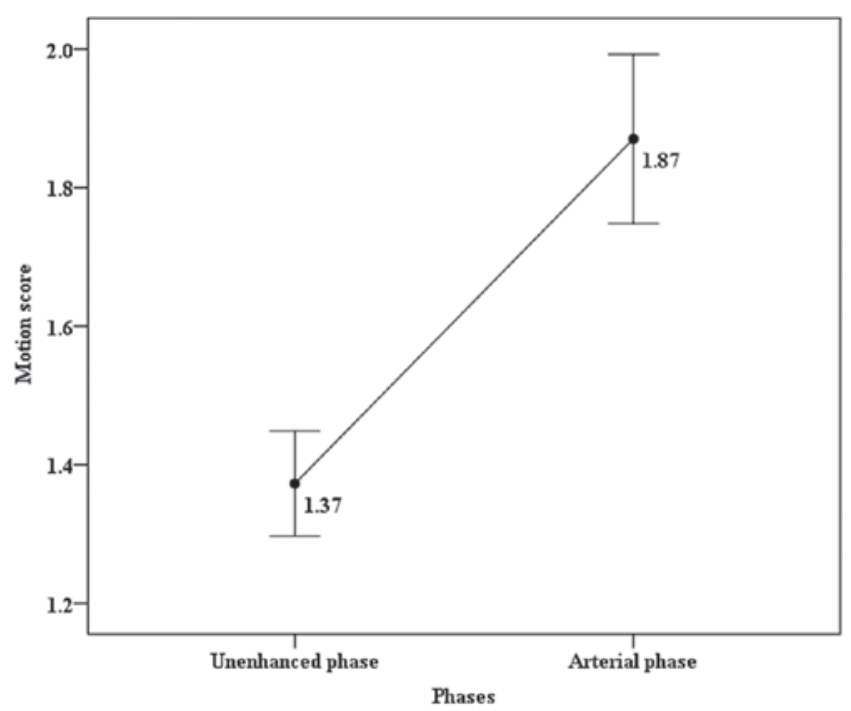

Figure 2. In single-VIBE group, the motion scores on arterial phase are significantly higher than those on unenhanced phase ( 1.87 vs. $1.37, \mathrm{P}<0.05)$.

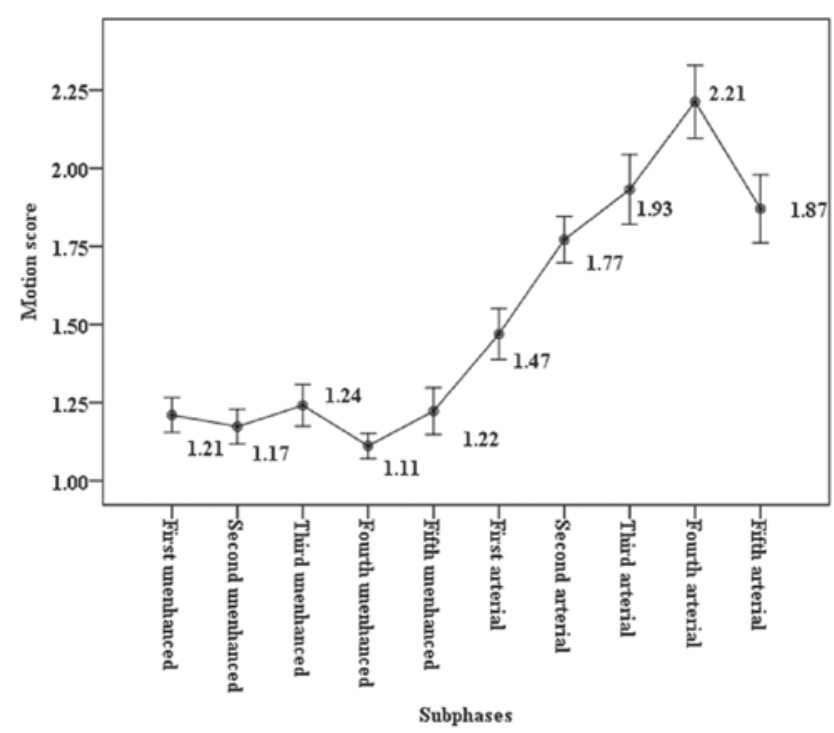

Figure 3. In 5-CDT-VIBE group, the motion scores on five unenhanced subphases tend to be stable, but among five arterial subphases, motion scores increase progressively up to the plateau at the fourth arterial phase, and then slowly decrease till to the fifth arterial phase.

agreement between two reviewers. Agreement was classified as poor (ICC, 0-0.40), fair to good (ICC, 0.40-0.75), or
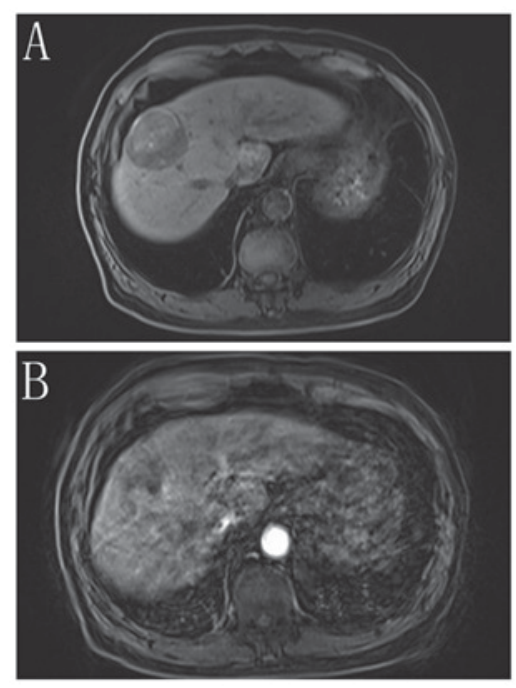

Figure 4. Typical imaging in single-VIBE group. A 53 years old man with HCC underwent Gd-EOB-DTPA-enhanced MR. The images show the patient without motion artifact on unenhanced phase with motion score of 1 (A), but on the arterial phase, severe motion artifact with the motion score of 5 can be observed (B), indicates presence of TSM. In this patient, only one arterial phase is obtained, causing an inadequate diagnostic performance for diagnosis.

excellent (ICC, $>0.75$ ). Differences in age and BMI between single-VIBE group and 5-CDT-VIBE group were compared using Mann-Whitney U Test. Results of Child-Pugh grading, gender, and volume of ascites grading between single-VIBE group and 5-CDT-VIBE group were assessed using $\chi^{2}$ test or Fisher exact test (if appropriate). Comparison of the above parameters was also performed in the overall study population, patients with TSM, and patients without TSM. Mean score between unenhanced phase and arterial phase was compared using Wilcoxon Rank Sum Test. All statistical analysis was performed under SPSS 19.0 software (SPSS, Inc., Chicago, IL, USA). $\mathrm{P}<0.05$ was considered to indicate a statistically significant difference.

\section{Results}

Study population. The patients' BMI and age between single-VIBE group and 5-CDT-VIBE group were compared, but were not found to be statistically significant $(20.1 \pm 2.5$ vs. $21.9 \pm 2.1, \mathrm{P}=0.14 ; 53.1 \pm 14.2$ vs. $59.8 \pm 19.4, \mathrm{P}=0.073$; respectively). However, in the overall study population, single-VIBE group, as well as 5-CDT-VIBE group, patients with TSM 


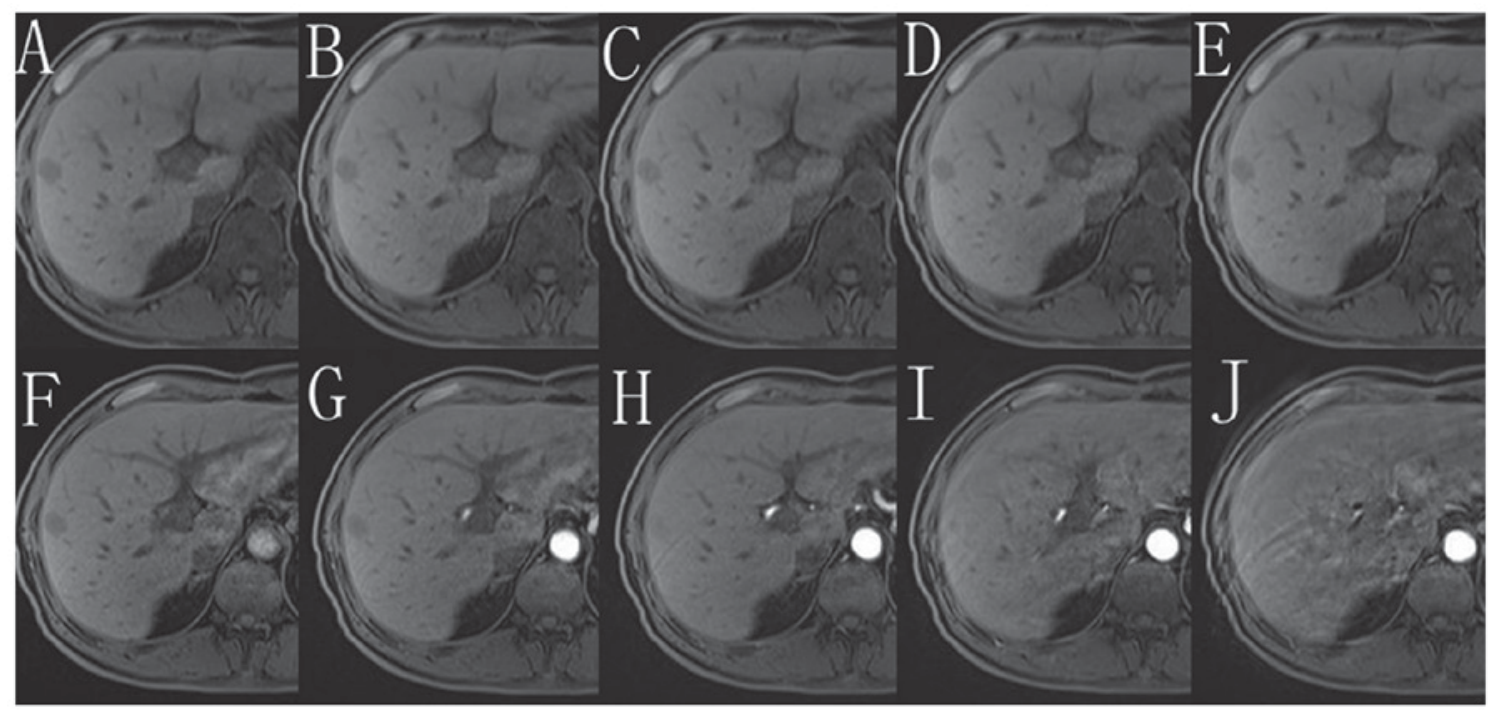

Figure 5. Typical imaging in 5-CDT-VIBE group. A 45 years old woman with HCC underwent Gd-EOB-DTPA-enhanced MR. The images show the patient without motion artifact (motion score is less than 2 in each image) among each five unenhanced subphases (A-E), while among five arterial subphases, the artifact can be observed, severely at the fifth one (motion score is 4), which indicates presence of TSM (F-J). A liver lesion is detected in all phases except the fifth arterial subphase due to the severe motion artifact. Despite of the severe motion artifact, the remaining arterial subphases are sufficient to provide adequate quality images.

showed significantly higher BMI and volume of ascites grading than those of patients without TSM, which might indicate that TSM more frequently occurs in patients with a higher BMI or ascites volume. The characteristics of patients were listed in Table III.

Consistency analysis. Reviewers' agreement of motion score calculation was evaluated by ICC. There was fair to good to excellent reproducibility of mean motion score in each phase between two reviewers, with the ICCs ranging from 0.68 (the third arterial phase) to 0.93 (the fifth unenhanced phase), indicating an acceptable inter-observer agreement. The detailed ICCs between two reviewers were listed in Table IV.

Comparison between two groups. In the overall study population, there were 216 patients in the single-VIBE technique group, and 162 patients in the 5-CDT-VIBE technique group. TSM occurred in 47 patients $(47 / 378,12.4 \%)$ in total, with 25 patients $(25 / 216,11.6 \%)$ in the single-VIBE group, and 22 patients $(22 / 162,13.6 \%)$ in the 5-CDT-VIBE group. The frequency rate of TSM between the two groups was not statistical significant (11.6 vs. $13.6 \%, \mathrm{P}>0.05$ ).

In the single-VIBE group, the motion score on arterial phase was significantly higher than those on unenhanced phase (1.87 vs. $1.37, \mathrm{P}<0.05)$. In the 5-CDT-VIBE group, motion scores on five arterial subphases $(1.47,1.77,1.93,2.21$ and 1.87 , respectively corresponding to the first, second, third, fourth, and fifth arterial phases) were significantly higher $(\mathrm{P}<0.05$ for all five comparisons) than those on five unenhanced subphases (1.21, 1.17, 1.24, 1.11 and 1.22 , respectively corresponding to the first, second, third, fourth, and fifth unenhanced phases). The motion score among the five arterial subphases increased progressively up to the peak at the fourth arterial phase, and then slowly decreased until the fifth arterial phase. The change of motion score is shown in Figs. 2 and 3. For patients with TSM in the 5-CDT-VIBE group, the highest motion scores occurred during the fourth arterial phase $(12 / 22,54.5 \%)$, the third arterial phase $(7 / 22,31.8 \%)$, and the fifth arterial phase $(3 / 22,13.7 \%)$, and 20 out of $22(20 / 22,90.9 \%)$ patients with TSM provided adequate arterial phase diagnostic information attributed to the remaining arterial subphases. Nevertheless, 0 of the 25 patients with TSM could provide adequate diagnostic imaging in the single-VIBE group. Figs. 4 and 5, respectively demonstrates typical imaging of the single-VIBE group compared with the 5-CDT-VIBE group. Fig. 4 shows a patient without motion artifact on unenhanced phase, but on arterial phase, severe motion artifact is observed, indicating the presence of TSM. In this patient, only one arterial phase was obtained, leading to inadequate image quality for diagnosis. Fig. 5 shows a patient without motion artifact on each of five unenhanced subphases, while among five arterial subphases, motion artifact can be observed, most severe at the fifth phase. Despite the severe motion artifact of the fifth arterial subphase, the remaining subphases are sufficient to provide adequate quality images.

\section{Discussion}

TSM on arterial phase imaging during Gd-EOB-DTPA acquisition has been frequently described (21-23). Since arterial phase enhancement is accepted as one of the diagnostic criteria for HCC in LI-RADS, it is crucial to provide optimal arterial phase imaging. In the present study, we confirm that 5-CDT-VIBE technique can provide sufficient diagnostic information on arterial phase for patients who display TSM during Gd-EOB-DTPA-enhanced MR. In a single-phase technique group, 0 of 25 patients with TSM obtained adequate image quality on arterial phase, in contrast however, in a 5-CDT-VIBE technique group, 20 out of 22 patients with TSM could provide adequate diagnostic information on arterial phase attributing to the other arterial subphases, which were artifact free. 
The prevalence of TSM related to Gd-EOB-DTPA administration has been reported to range from 2 to $19 \%(15,17,20-25)$. In the present study, prevalence of TSM is $12.4 \%$ (47/378) in the overall study population, which shows a lower rate than the studies demonstrated by Haradome et al (20 of 108 patients, 19\%) (24) and Davenport et al (17 of 99 patients, 17\%) (15). Possible reason for this discrepancy is the total volume of contrast agent administered. In previous studies, 10 to $20 \mathrm{ml}$ total volume of contrast agent were utilized for examination, while in our study a less volume was used $(0.1 \mathrm{ml} / \mathrm{kg}$ body weight). Higher doses have previously been reported to correlate with TSM (17). Likewise, several studies reveal that the body weight of the patient is an independent risk factor for TSM (22). We compare five patient characteristics such as BMI, age, Child-Pugh grading, gender, and volume of ascites gradingbetween patients with TSM and without TSM. The results reveal that TSM more frequently occurs in patients with elevated BMI or ascites volume grading. Because we use a body weight-tailored dose administration strategy, in those of patients with higher BMI or volume of ascites, they may have a higher degree of adiposity or body weight, as a result more contrast dose received.

Interestingly, the results of this study also demonstrate that in 22 patients with TSM among the 5-CDT-VIBE group, the highest motion score occurs during the fourth arterial phase (mean motion score is $2.21,12 / 22,54.5 \%$ ), the third arterial phase (mean motion score of $1.93,7 / 22,31.8 \%$ ), and the fifth arterial phase (mean motion score of $1.87,3 / 22,13.7 \%$ ). In our study, using the 5-CDT-VIBE technique, initial arterial phase starts at a standard timing of $18 \mathrm{sec}$ following the administration of contrast with a temporal resolution of $2.6 \mathrm{sec}$. The fourth arterial phase starts at 25.8 to $28.4 \mathrm{sec}$. In a cohort study conducted by Pietryga et al showed the highest motion score occurred at the third arterial phase from the timing of 30 to $37.5 \mathrm{sec}$ after initiation of contrast agent injection (25). This discrepancy is most likely due to the difference in temporal resolution. In their study, the temporal resolution in each arterial subphase acquisition is $7.5 \mathrm{sec}$, but in our study, the temporal resolution is only $2.6 \mathrm{sec}$. As it has been reported that higher temporal resolution affords more accurate measurement of peak and dynamic changes in signal intensity (26), our study shows an early arrival of the highest motion score.

In Pietryga et al study, they used a triple arterial subphase technique to reduce the effect of TSM on arterial phase with Gd-EOB-DTPA administration (25). However, this technique had some disadvantages, such as reduction of spatial resolution and a relatively thicker slice thickness, which may reduce the signal to noise ratio of each individual phase. In the present study, we use a 5-CDT-VIBE technique to obtain 5 arterial subphases with a single breath holding. 5-CDT-VIBE is a newly developed technique, which can achieve robust contrast-enhanced imaging of the liver with high temporospatial resolution $(19,27)$.

Our study has some limitations. Firstly, this study is retrospectively designed. We did not randomly make the decision for which technique the patient would receive. Secondly, only the patient with liver lesions is enrolled, other liver diseases such as liver cirrhosis is not included in the study. Liver cirrhosis may have an impact on patients' breath holding. Thus, selection bias may have been presented. Thirdly, we do not compare liver lesion detection between single-VIBE group and 5-CDT-VIBE group. A study conducted by Kazmierczak et al reveals that 5-CDT-VIBE technique can significantly improve the detection and differential diagnosis of hypervascular FLLs (28). We have not assessed the utility of 5-CDT-VIBE technique in patients with or without TSM for FLLs detection. Thirdly, as breath-holding is important in the reduction of respiratory motion artifacts (29), we did not keep a unified breath-holding time in two groups. The reason may be that a fixed breath-holding time of $20 \mathrm{sec}$ is used in 5-CDT-VIBE technique, while for single-vibe technique, the breath-holding time is unfixed. Finally, we have not evaluated the nonarterial post-contrast image and the adequacy of arterial phase timing. Pietryga et al (25) reviewed the motion artifact by using an additional scoring system to evaluate the adequacy of late hepatic arterial phase timing, which may have higher accuracy.

In conclusion, Gd-EOB-DTPA related TSM mostly occurs between the 25.8 to $28.4 \mathrm{sec}$ (the fourth arterial phase) following contrast administration. We conclude that 5-CDT-VIBE technique can provide images with diminished artifact in patients with TSM.

\section{Competing interests}

The authors declare that they have no competing interests

\section{References}

1. Nishie A, Kakihara D, Asayama Y, Ushijima Y, Takayama Y, Fujita N, Shimamoto D, Shirabe K, Hida T and Honda H: Detectability of hepatocellular carcinoma on gadoxetic acid-enhanced MRI at $3 \mathrm{~T}$ in patients with severe liver dysfunction: Clinical impact of dual-source parallel radiofrequency excitation. Clin Radiol 70: 254-261, 2015.

2. Verloh N, Utpatel K, Haimerl M, Zeman F, Fellner C, Fichtner-Feigl S, Teufel A, Stroszczynski C, Evert M and Wiggermann P: Liver fibrosis and Gd-EOB-DTPA-enhanced MRI: A histopathologic correlation. Sci Rep 5: 15408, 2015.

3. Esterson YB, Flusberg M, Oh S, Mazzariol F, Rozenblit AM and Chernyak V: Improved parenchymal liver enhancement with extended delay on Gd-EOB-DTPA-enhanced MRI in patients with parenchymal liver disease: Associated clinical and imaging factors. Clin Radiol 70: 723-729, 2015.

4. Haimerl M, Verloh N, Fellner C, Zeman F, Teufel A, Fichtner-Feigl S, Schreyer AG, Stroszczynski C and Wiggermann P: MRI-based estimation of liver function: Gd-EOB-DTPA-enhanced T1 relaxometry of 3T vs. the MELD score. Sci Rep 4: 5621, 2014.

5. Hwang J, Kim YK, Jeong WK, Choi D, Rhim H and Lee WJ: Nonhypervascular hypointense nodules at gadoxetic acid-enhanced MR imaging in chronic liver disease: Diffusion-weighted imaging for characterization. Radiology 276: 137-146, 2015.

6. Tse JR, Naini BV, Lu DS and Raman SS: Qualitative and quantitative gadoxetic acid-enhanced MR imaging helps subtype hepatocellular adenomas. Radiology 279: 118-127, 2016.

7. Xiao YD, Paudel R, Liu H, Zhang B, Ma C and Zhou SK: Gadolinium ethoxybenzyl diethylenetriamine pentaacetic acid-enhanced magnetic resonance imaging: A potential utility for the evaluation of regional liver function impairment following transcatheter arterial chemoembolization. Oncol Lett 9: 1191-1196, 2015.

8. Kubota K, Tamura T, Aoyama N, Nogami M, Hamada N, Nishioka A and Ogawa Y: Correlation of liver parenchymal gadolinium-ethoxybenzyl diethylenetriaminepentaacetic acid enhancement and liver function in humans with hepatocellular carcinoma. Oncol Lett 3: 990-994, 2012.

9. Tamada T, Ito K, Sone T, Kanki A, Sato T and Higashi H: Gd-EOB-DTPA enhanced MR imaging: Evaluation of biliary and renal excretion in normal and cirrhotic livers. Eur J Radiol 80: e207-e211, 2011. 
10. Xiao YD, Paudel R, Liu J, Ma C, Zhang ZS and Zhou SK: MRI contrast agents: Classification and application (Review). Int $\mathrm{J}$ Mol Med 38: 1319-1326, 2016.

11. Haradome H, Grazioli L, Al Manea K, Tsunoo M, Motosugi U, Kwee TC and Takaraha T: Gadoxetic acid disodium-enhanced hepatocyte phase MRI: Can increasing the flip angle improve focal liver lesion detection? J Magn Reson Imaging 35: 132-139, 2012.

12. Ahn SS, Kim MJ, Lim JS, Hong HS, Chung YE and Choi JY: Added value of gadoxetic acid-enhanced hepatobiliary phase MR imaging in the diagnosis of hepatocellular carcinoma. Radiology 255: 459-466, 2010.

13. Xiao YD, Ma C, Liu J, Li HB, Zhang ZS and Zhou SK: Evaluation of hypointense liver lesions during hepatobiliary phase MR imaging in normal and cirrhotic livers: Is increasing flip angle reliable? Sci Rep 6: 18942, 2016.

14. Aran S, Shaqdan KW and Abujudeh HH: Adverse allergic reactions to linear ionic gadolinium-based contrast agents: Experience with 194, 400 injections. Clin Radiol 70: 466-475, 2015.

15. Davenport MS, Viglianti BL, Al-Hawary MM, Caoili EM, Kaza RK, Liu PSC, Maturen KE, Chenevert TL and Hussain HK Comparison of acute transient dyspnea after intravenous administration of gadoxetate disodium and gadobenate dimeglumine: Effect on arterial phase image quality. Radiology 266: 452-461, 2013.

16. Li H, Xiao Y, Wang S, Li Y, Zhong X, Situ W, Xiao E and Zhang Z: TWIST-VIBE five-arterial-phase technology decreases transient severe motion after bolus injection of Gd-EOB-DTPA Clin Radiol 72: 800 e1-800.e6, 2017.

17. Davenport MS, Bashir MR, Pietryga JA, Weber JT, Khalatbari S and Hussain HK: Dose-toxicity relationship of gadoxetate disodium and transient severe respiratory motion artifact. AJR Am J Roentgenol 203: 796-802, 2014.

18. Fujinaga $Y$, Ueda H, Kitou Y, Tsukahara Y, Sugiyama $Y$ and Kadoya M: Time-intensity curve in the abdominal aorta on dynamic contrast-enhanced MRI with high temporal and spatial resolution: Gd-EOB-DTPA versus Gd-DTPA in vivo. Jpn J Radiol 31: 166-171, 2013

19. Michaely HJ, Morelli JN, Budjan J, Riffel P, Nickel D, Kroeker R, Schoenberg SO and Attenberger UI: CAIPIRINHA-Dixon-TWIST (CDT)-volume-interpolated breath-hold examination (VIBE): A new technique for fast time-resolved dynamic 3-dimensional imaging of the abdomen with high spatial resolution. Invest Radiol 48: 590-597, 2013.

20. Yoo JL, Lee CH, Park YS, Kim JW, Lee J, Kim KA, Seol HY and Park CM: The short breath-hold technique, controlled aliasing in parallel imaging results in higher acceleration, can be the first step to overcoming a degraded hepatic arterial phase in liver magnetic resonance imaging: A prospective randomized control study. Invest Radiol 51: 440-446, 2016.
21. Bashir MR, Castelli P, Davenport MS, Larson D, Marin D, Hussain HK and Jaffe TA: Respiratory motion artifact affecting hepatic arterial phase MR imaging with gadoxetate disodium is more common in patients with a prior episode of arterial phase motion associated with gadoxetate disodium. Radiology 274 : 141-148, 2015.

22. Hayashi T, Saitoh S, Tsuji Y, Takahashi J, Tagaya N, Hiramoto M, Fukuzawa K, Tano M, Miyati T and Kumada H: Influence of gadoxetate disodium on oxygen saturation and heart rate during dynamic contrast-enhanced MR imaging. Radiology 276: 756-765, 2015.

23. Motosugi U, Bannas P, Bookwalter CA, Sano K and Reeder SB: An investigation of transient severe motion related to gadoxetic acid-enhanced MR imaging. Radiology 279: 93-102, 2016.

24. Haradome H, Grazioli L, Tsunoo M, Tinti R, Frittoli B, Gambarini S, Morone M, Motosugi U and Colagrande S: Can MR fluoroscopic triggering technique and slow rate injection provide appropriate arterial phase images with reducing artifacts on gadoxetic acid-DTPA (Gd-EOB-DTPA)-enhanced hepatic MR imaging? J Magn Reson Imaging 32: 334-340, 2010.

25. Pietryga JA, Burke LMB, Marin D, Jaffe TA and Bashir MR: Respiratory motion artifact affecting hepatic arterial phase imaging with gadoxetate disodium: Examination recovery with a multiple arterial phase acquisition. Radiology 271: 426-434, 2014.

26. Fujinaga Y, Ohya A, Tokoro H, Yamada A, Ueda K, Ueda H, Kitou Y, Adachi Y, Shiobara A, Tamaru N, et al: Radial volumetric imaging breath-hold examination (VIBE) with k-space weighted image contrast (KWIC) for dynamic gadoxetic acid (Gd-EOB-DTPA)-enhanced MRI of the liver: Advantages over cartesian VIBE in the arterial phase. Eur Radiol 24: 1290-1299, 2014.

27. Budjan J, Ong M, Riffel P, Morelli JN, Michaely HJ, Schoenberg SO and Haneder S: CAIPIRINHA-Dixon-TWIST (CDT)-volume-interpolated breath-hold examination (VIBE) for dynamic liver imaging: comparison of gadoterate meglumine, gadobutrol and gadoxetic acid. Eur J Radiol 83: 2007-2012, 2014.

28. Kazmierczak PM, Theisen D, Thierfelder KM, Sommer WH, Reiser MF, Notohamiprodjo $M$ and Nikolaou $K$ : Improved detection of hypervascular liver lesions with CAIPIRINHA-Dixon-TWIST-volume-interpolated breath-hold examination. Invest Radiol 50: 153-160, 2015.

29. Gutzeit A, Matoori S, Froehlich JM, von Weymarn C, Reischauer C, Kolokythas O, Goyen M, Hergan K, Meissnitzer M, Forstner R, et al: Reduction in respiratory motion artefacts on gadoxetate-enhanced MRI after training technicians to apply a simple and more patient-adapted breathing command. Eur Radiol 26: 2714-2722, 2016. 